\title{
Correspondence
}

\section{Flawed policies}

Sir: Our local experience suggests that national and local policies to reduce risks of suicide and harm to others by psychiatric patients may be doing more harm than good.

We suspect that the situation in York is not untypical. A recent survey showed that, of the 63 patients in our three acute admission wards, half were under a compulsory order and eight were on level one observation. The number of patients on level one observation has on occasion been much higher. Nursing staff on duty caring for these patients numbered between 14 and 16 . With eight of this nursing complement fully engaged in one-to-one observation, then only six to eight nurses remain available to care for the remaining 55 patients. The increasing paperwork relative to compulsory orders, Care Programme Approach and risk management means that, in practice. there are even fewer nurses available to these 55 patients.

The high investment of resources in high-risk patients driven by national guidance and local policy with respect to level one observation. could be increasing the risk to patients and the public. It is another example of the epidemiological paradox. The high-risk group on level one observation pose a lesser risk, because there are few of them, than the majority at lower risk simply because there are many more of them. This has been tragically illustrated recently by a suicide and two serious assaults on others which were perpetrated by patients not on level one observation. It is not at all surprising that the half-dozen nurses left to try to care for the $\mathbf{5 5}$ patients not on one-to-one observation sometimes fail to detect sudden deteriorations in mental state.

So, it is not just that the policy of level one observation is transforming the culture of psychiatric wards from therapeutic to custodial, which may in itself raise risks, it is also that the flexibility of nursing staff to monitor and respond to the needs of all patients under their care is so reduced as to give a lot to the few and virtually nothing to the majority. It would be a travesty of the truth to pretend that six nurses can be sensitive to the general needs of 55 patients. Fourteen skilled and flexibly working nurses might just be able to manage risks better by keeping in touch with all of their charges most of the time.

However, the national trend is of increasing the proportion of untrained/trained nursing staff on acute psychiatric wards. This has come about partly as a way of replacing student nurses but also to maintain nursing numbers with tighter budgets. The national average now is 60 trained staff to 40 untrained staff. But in practice day-today levels are nearer 50:50 as untrained supply staff are often employed. Perhaps this trend needs to be reversed, if it means that half the nurses on duty are not capable of making the kind of mental state assessments necessary to manage risk flexibly.

Those nursing staff who are deployed to do the unrewarding job of level one observation report feeling tense and uncomfortable with this task. If the nurse feels tense with such close and continuous proximity, what does it do to someone in an acute psychotic state? Could level one observation make patients feel worse?

We asked the Mental Health Commission for examples across the country of exemplary practice in the management of acute psychiatric wards. They would not give us an answer.

Clearly, more nursing resource is highly desirable for managing the increasingly disturbed population of patients in acute psychiatrc wards. If more resource is not forthcoming, we suggest that psychiatric wards would be more therapeutic and safer if nurses are encouraged to exercise their skills in observation and care flexibly, rather than being bound by rigid observation procedures which seem to be defeating the objectives they were established to achieve.

R. D. ADAMS. Consultant Psychiatrist and Clinical Director and P. F. KENNEDY, Chief Executive, York Health Services Trust. Chief Executive's Office. Bootham Park, York YO3 7BY

\section{Psychiatric in-patients of no fixed abode}

Sir: Mental illness among homeless people has long been perceived as a significant health problem (Berry \& Orwin, 1966). Homelessness among the psychiatric in-patient population has been reported to be as high as $10 \%$, or 10 times the rate of homelessness among the general population (Neville \& Masters, 1990). Homeless males have been shown to be socially less stable than their female counterparts, and to out number them by a factor of three to one (Herzberg, 1987). 
In order to inform future service planning, we undertook a retrospective review of homelessness among the mentally ill admitted to an innerLondon unit with a catchment area population of 170000 of whom approximately $1 \%$ were homeless (Neville \& Masters, 1990).

The study took the form of a retrospective case note study of all admissions to the unit over a 30month period, 1 January 1992 to June 1994 inclusive. Patients were defined as being homeless or of no fixed abode if they were homeless at the time of admission and had no home to return to on discharge (Herzberg, 1987). For the purposes of statistical analysis, consultant assigned diagnoses using International Classification of Diseases (ICD-10) were summated into the broad categories of: schizophrenia, affective disorders, personality disorders, alcohol misuse, other drug misuse, other diagnoses.

A total of 133 in-patients (101 males) of no fixed abode were identified; a mean annual rate of 53 per year, or $11 \%$ of all admissions annually. Eighteen $(18 \%)$ males and $9(28 \%)$ females were referred by the police; $9(9 \%)$ males and no females were admitted via the prison services. Twenty-seven (27\%) males and 11 (34\%) females of no fixed abode were detained under the Mental Health Act (1983).

Mean age of no fixed abode patients on admission was 33 years (s.d.11) and was similar for both genders. Some $80 \%$ of both males and females of no fixed abode had never been married: $53(52 \%)$ males and $18(56 \%)$ females had contact with a family member within the previous year and $2(2 \%)$ males and no females were currently employed. Approximately onethird of males and females were currently registered with a general practitioner.

The diagnostic profiles of males and females were strikingly similar: schizophrenia was the most common diagnosis, $42(42 \%)$ males and 10 (34\%) females; followed by alcohol misuse in the case of males, $19(19 \%)$ and both alcohol misuse and personality disorders in the case of females - each accounting for 5 (16\%). Affective disorders accounted for a further $13 \%$ in both sexes. Diagnosis was not available in $9 \%(9$ males and 3 females).

In the unit under study, the proportion of inpatients who were of no fixed abode was of a high order and in keeping with a recent report from a catchment area of similar demographic characteristics (Neville \& Masters, 1990). Similar to previous studies, schizophrenia was the most common diagnosis (Berry \& Orwin, 1966; Herzberg, 1987). Contrary to Herzberg's findings (1987), there was little evidence that females were socially more stable than males.

Community psychiatry faces a further challenge in meeting the needs of this small but substantial subgroup of vulnerable patients.
BERRY, C \& Orwin, A. (1966) No fixed abode: a survey of mental hospital admissions. British Journal of Psychiatry, 112. 1019-1025.

HERZBERG. J. L. (1987) No fixed abode: a comparison of men and women admitted to an east London psychiatric hospital. British Journal of Psychiatry, 160, 621-627.

Nenile. M. \& MASTERS, D. (1990) A Report of Inpatient Activity on Mental Illness Wards at Guy's Hospital for the Full Year 1989. London: Lewisham and North Southwark Mental Health Executive.

MIRIAM O'DOHERTY, St Vincent's Hospital, Convent Avenue, Richmond Road, Dublin 3; VIV MAK and MARIOS PIERIDES, Lambeth Healthcare NHS Trust, Landor Road, London SW9 9NT

\section{High dose antipsychotic medication}

Sir: The findings of Sillifant et al (Psychiatric Bulletin, October 1997, 21, 612-617) that four out of 11 patients on high dose antipsychotic medication have low white cell counts should not cause alarm or surprise. A large-scale survey by Pisciotta (1971) of patients taking phenothiazines was reported 26 years ago. Of 6200 patients, 2000 developed moderate leukopenia. This was found to be a transient phenomenon. These results may help to alleviate the anxiety caused by minimally abnormal results reported by Sillifant and her colleagues.

Pisciotta, V. A. (1971) Drug-induced leukopenia and aplastic anaemia. Clinical Pharmacology and Therapeutics, 12, 13-43.

J. WAITE, Consultant Psychogeriatrician, Nottingham Healthcare NHS Trust, Health Care of the Elderly, The Courtyard, East Block, University Hospital, Nottingham NG7 $2 U H$.

\section{Definition of 'haltlose'}

Sir: While involved in the undergraduate teaching programme in psychiatry, I was asked by one of my students to explain the term 'haltlose' as it appears in the ICD-10 Classification of Mental and Behavioural Disorders (World Health Organization, 1992). This term can be found in the category of 'Specific Personality Disorders' (F60) where it is included under the heading of 'Other Specific Personality Disorders' (F60.8). This rubric is used to classify: 'A personality disorder that fits none of the specific rubrics F60.0 F60.7', and includes the terms: eccentric, 'haltlose' type, immature, narcissistic, passiveaggressive, and psychoneurotic personality (disorder). While the other terms in this rubric are in relatively common usage, I was not clear as to the meaning of the term 'haltlose' as used in psychiatric practice or otherwise.

A literature search was then carried out, however there were no records of 'haltlose' on 